\title{
Gallstone Ileus: An Uncommon Cause of Acute Abdomen
}

\author{
Rachida Saouab ${ }^{1}$, Fatima Zahra Rhouni ${ }^{2}$, Mohammed Mahi' ${ }^{2}$ Hassan Boumdien ${ }^{2}$, Touriya Amil', \\ Jamal El Fenni ${ }^{2}$
}

${ }^{1}$ Department of Radiology, Military Hospital, Faculty of Medicine and Pharmacy, Cadi Ayyad University, Marrakech, Morocco

${ }^{2}$ Department of Radiology, Military Hospital, Faculty of Medicine and Pharmacy, Mohammed V University, Rabat, Morocco

\section{Email address:}

rachida_sa@yahoo.fr(R. Saouab)

\section{To cite this article:}

Rachida Saouab, Fatima Zahra Rhouni, Mohammed Mahi, Hassan Boumdien, Touriya Amil, Jamal El Fenni. Gallstone Ileus: An Uncommon Cause of Acute Abdomen. International Journal of Medical Imaging. Vol. 7, No. 1, 2019, pp. 25-28. doi: 10.11648/j.ijmi.20190701.14

Received: March 5, 2019; Accepted: April 26, 2019; Published: May 17, 2019

\begin{abstract}
Gallstone ileus is a rare cause of acute abdomen, representing less than $1 \%$ of intestinal obstruction. It is due to mechanical small bowel obstruction (SBO) by a migrated gallstone. we report this observation to illustrate the imaging findings especially in CT scan. A 62-year-old woman was admitted to emergency for acute bowel obstruction. The CT scan has confirmed jejunal bowel distension with fluid levels upstream endoluminal formation which was rounded and well limited, measuring $5 \mathrm{~cm}$ of long axis. This training was slightly hyperdense with hypodense areas of fat density. Moreover, there was aerobilia with multiple fistulas between the duodenum (D1) and gallbladder, which was empty. The triad of aerobilia, small bowel obstruction and migrant gallstone was suggestive of gallstone ileus diagnosis. The patient was operated with good evolution. Gallstone ileus is an uncommon presentation of gallstone disease. It is frequently observed in elderly patients with a history of cholelithiasis or cholecystitis. Early diagnosis is crucial and would avoid morbidity and mortality related to this disease. CT scan shows the classical triad of Rigler with pneumobilia, an ectopic stone and mechanical ileus. CT may improve the diagnosis providing important information regarding the exact number, size, and location of ectopic stones and the site of intestinal obstruction or direct visualization of a biliary-enteric fistula, to help clinicians in the therapeutic management of patients.
\end{abstract}

Keywords: Gallstone, Ileus, Bowel Obstruction, Pneumobilia, Acute Abdomen, CT Scan

\section{Introduction}

Gallstone ileus is an uncommon cause of bowel obstruction which mainly affects the elderly population. It is a rare and serious complication of chronic cholecystitis and occurs when a gallstone passes into the small bowel and usually impacts at the ileocaecal valve. The associated mortality is estimated to be up to $30 \%$ [1]. This high mortality is attributed to comorbidities, particularly cardiovascular, respiratory, and endocrine (diabetes and obesity), or delayed diagnosis [2, 3]. Diagnostic imaging plays an important role in the management of patients. We report a case of a woman presented with jejunal obstruction due to migrant gallstone in order to remember the CT scan findings.

\section{Case Report}

A 62-year-old woman was admitted to emergency with acute abdominal pain and vomiting. Abdominal history was significant for gallstone. Physical examination revealed an afebrile patient with diffuse abdominal tenderness more marked at the right upper quadrant. The laboratory tests showed leukocytosis at $13000 / \mu 1$ with slight increase in Creactive protein (CRP) and creatinine. Abdominal ultrasound found aerobilia with distended small bowel loops. Additional computed tomography scan (CT) was requested. It was made without injection of contrast material because of kidney failure. The CT scan has confirmed aerobilia and presence of multiple fistulas between the duodenum (D1) and gallbladder, which was empty, with the presence of stones in the cystic duct (figure 1). 


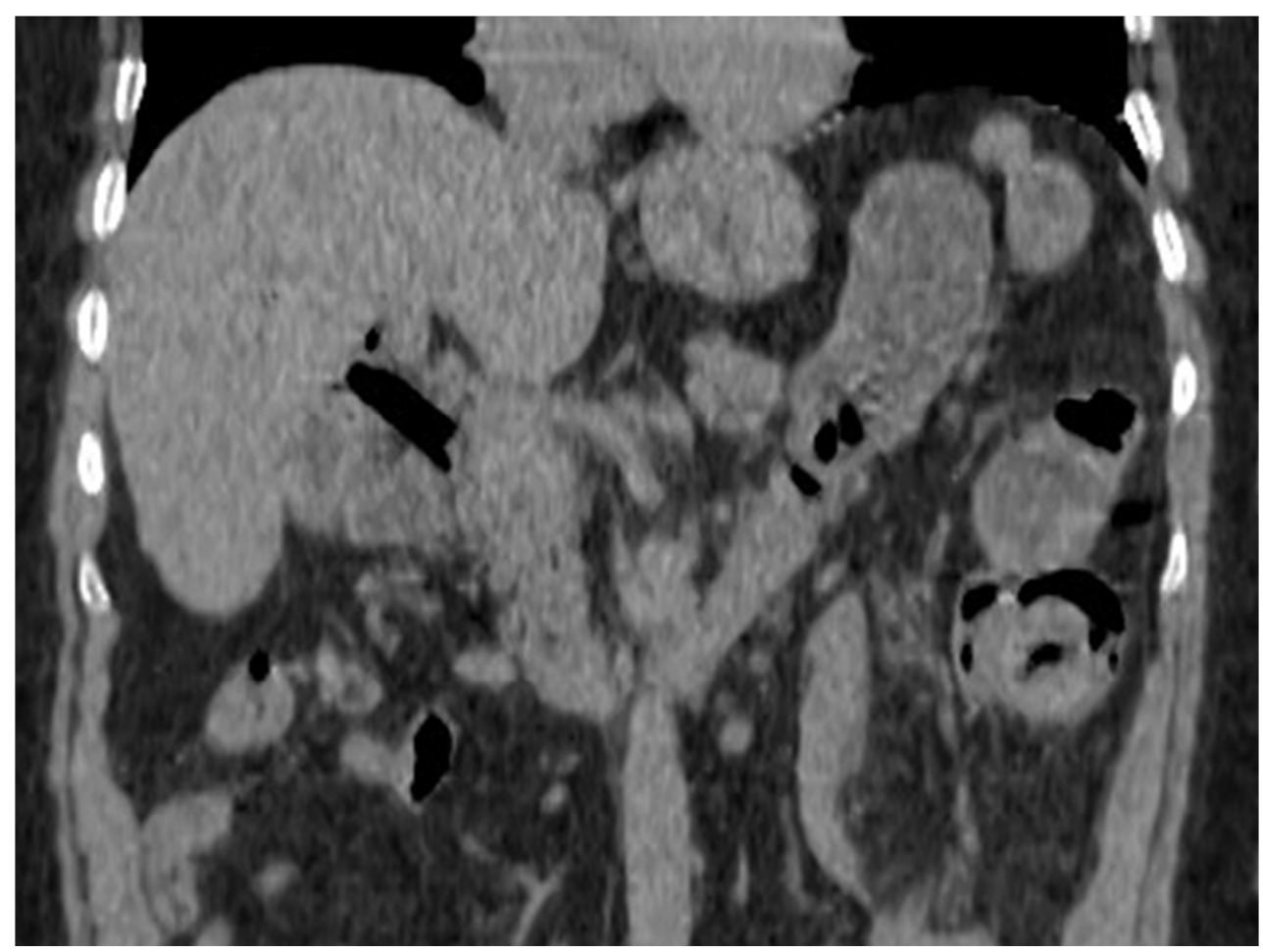

Figure 1. Coronal reconstruction computed tomography image without IV contrast injection reveals pneumobilia with multiple fistulas between the duodenum (D1) and gallbladder.

Moreover, there was small bowel distension with fluid levels upstream endoluminal formation which was rounded and well limited, measuring $5 \mathrm{~cm}$ of long axis. This training was slightly hyperdense with hypodense areas of fat density suggesting a migrant gallstone by the biliary- enteric fistula (figure 2).

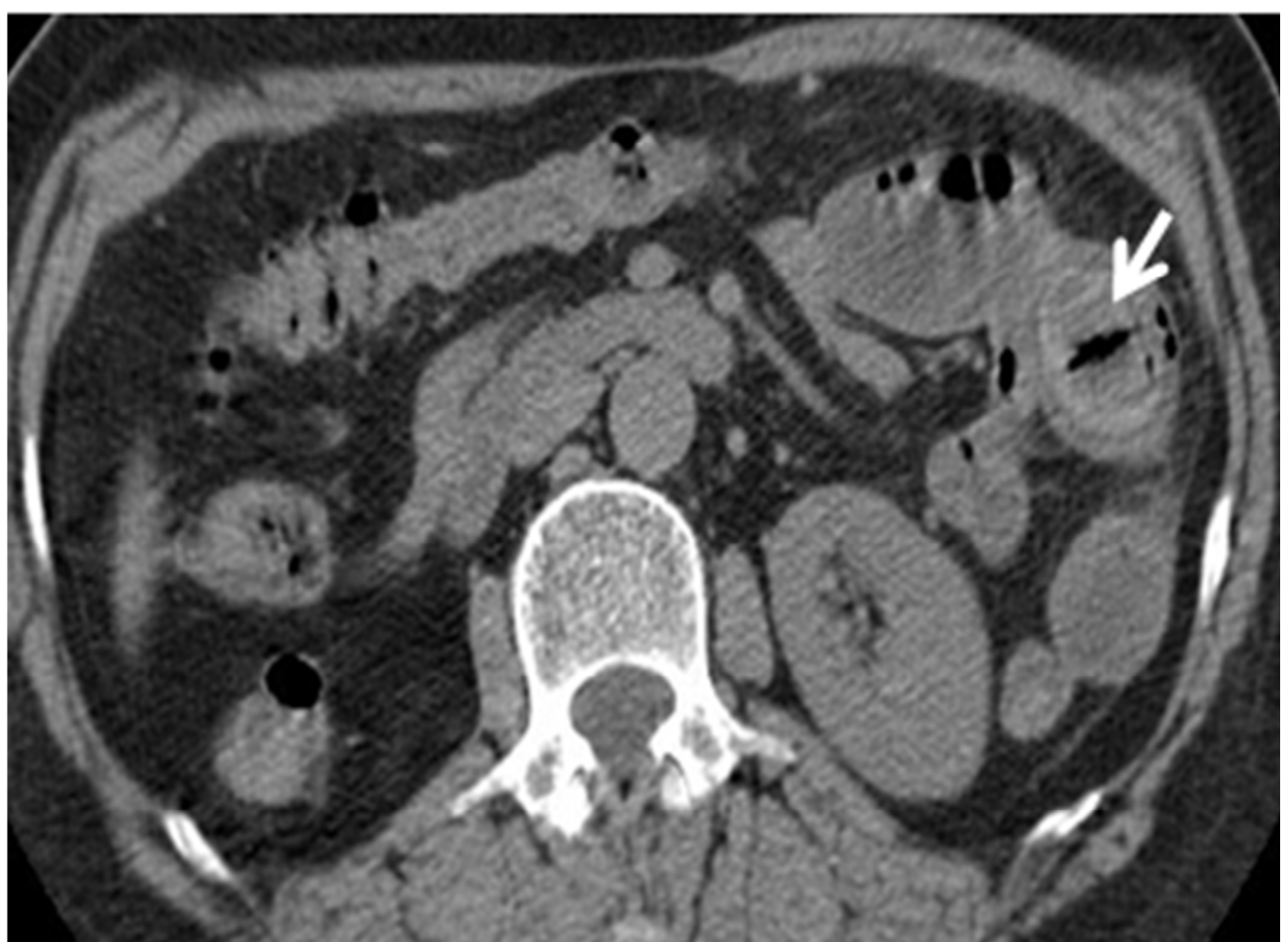

Figure 2. Axial computed tomography image without IV contrast injection reveals jejunal distension with endoluminal gallstone, measuring $5 \mathrm{~cm}$ of long axis. It was slightly hyperdense with hypodense areas of fat density.

The gallstone was radiotransparent at topogram (figure 3). 


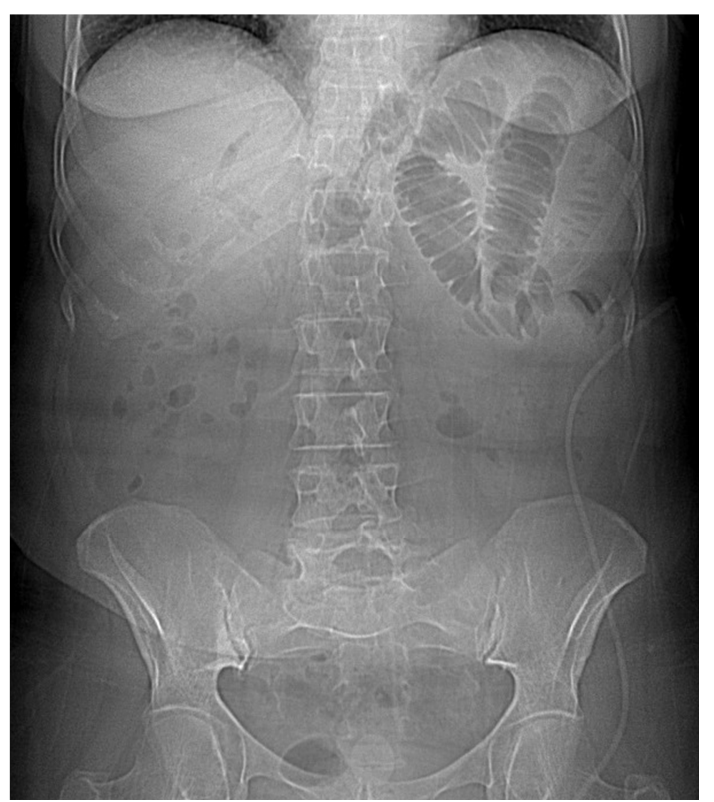

Figure 3. CT scan topogram reveals jejunal distension at the left hypochondrium without individualization of gallstone which is radiotransparent.

Ileum and colon were collapsed. The triad of aerobilia, small bowel obstruction and migrant gallstone was suggestive of gallstone ileus diagnosis. The patient was operated with good evolution.

\section{Discussion}

Gallstone ileus is an unusual complication of cholelithiasis, occurring in less than 0.5 percent of patients. It represents only 1 to $4 \%$ of small bowel obstruction caused by an impaction of a gallstone within the lumen of the small intestine via a cholecysto-enteric fistula. Large stones, greater than $2.5 \mathrm{~cm}$ in diameter, are thought to predispose to fistula formation by gradual erosion through the gallbladder fundus. Sixty percent are cholecystoduodenal fistulas, but cholecystocolonic and cholecystogastric fistulas can also result in gallstone ileus $[4,5]$. Common places for gallstones include the ileum and ileocecal valve due to the anatomical narrow lumen in $60 \%$ of cases, jejunum in up to $16 \%$, stomach in $15 \%$, and colon (gallstone coleus) in $2-8 \%$ of cases $[6,7]$.

The average age of patients with gallstone ileus is 70 years, with the youngest reported patient being 13 years of age. Women are 3 to 16 times more likely to be affected [8].

Typically, patients have a long history of recurrent right upper quadrant pain. The acute presentation of gallstone ileus is that of a small bowel obstruction, with colicky abdominal pain, abdominal distension and vomiting. Jaundice has been found in only $15 \%$ of patients or less. Upper gastrointestinal bleeding, secondary to duodenal erosion caused by the offending gallstone, with hematemesis and melena, can be seen in $15 \%$ and $7 \%$, respectively $[9,10]$.

The diagnosis of gallstone ileus is difficult and usually depends on the imaging findings. The classic Rigler's triad includes mechanical bowel obstruction, pneumobilia, and presence of an ectopic gallstone within the bowel lumen [11]. Plain abdominal radiographic films usually show nonspecific findings, because only $10 \%$ of gallstones are sufficiently calcified [12]. CT is the gold standard for positive diagnosis. The overall sensitivity, specificity and accuracy of CT in diagnosing gallstone ileus is around $93 \%$, $100 \%$; and $99 \%$, respectively. It allows detection of the stone whatever the density, its exact location and direct visualization of the biliary-enteric fistula. Typically, stones are large and measure several centimeters $(2-3 \mathrm{~cm})$. It is important to look for free fluid, free gas, portal venous gas, or mural gas, as signs of more advanced disease and poorer prognosis [13-16].

Gallstone ileus usually requires emergency surgery to relieve intestinal obstruction with the removal of the stone (enterolithotomy) and repair of the choledochoenteric fistula, accompanied by a cholecystectomy $[17,18]$.

Any delay in diagnosis and treatment may lead to serious complications such as electrolyte imbalance, ischemic lesions, ulcerations of the bowel, abscess formation, and, occasionally, free perforation and peritonitis. The morbidity and mortality rate of gallstone ileus remain very high, partly because of misdiagnosis and delayed diagnosis and otherwise because of the age-related co- morbidities of the afflicted patients [19].

\section{Conclusion}

Gallstone ileus is a frequently misdiagnosed clinical entity. Early diagnosis is crucial and allows earlier therapy and would avoid morbidity and mortality related to this disease. The use of radiological imaging is invaluable in the diagnosis of gallstone ileus. In fact, CT scanning is a powerful and gold-standard tool to diagnose the condition and to guide its management. The combination of pneumobilia and gastrointestinal obstruction should suggest the diagnosis of gallstone ileus. In addition to the Rigler radiologic triad, CT allows detection of the exact location of the ectopic stones, the site of obstruction and direct visualization of the biliaryenteric fistula.

\section{References}

[1] Liisa Chang, Minna Chang, Hanna M. Chang, Aina I. Chang, Fuju Chang. Clinical and radiological diagnosis of gallstone ileus: a mini review. Emerg Radiol (2018) 25:189-196

[2] Halabi WJ, Kang CY, Ketana N, Lafaro KJ, Nguyen VQ, Stamos MJ, Imagawa DK, Demirjian AN Surgery for gallstone ileus: a nationwide comparison of trends and outcomes. Ann Surg (2014) 259(2):329-335.

[3] Bass G, Gilani SN, Walsh TN Validating the 5Fs mnemonic for cholelithiasis: time to include family history. Postgrad Med J (2013) 89(1057):638-641.

[4] Farrell I, Turner P. A simple case of gallstone ileus? J Surg Case Rep 2015: rju148. 
[5] Ploneda-Valencia CF, Gallo-Morales M, Rinchon C, NavarroMuñiz E, Bautista-López CA, de la Cerda-Trujillo LF et al Gallstone ileus: an overview of the literature. Rev Gastroenterol (2017) Mex 82(3):248-254.

[6] Dai XZ, Li GQ, Zhang F, Wang XH, Zhang CY. Gallstone ileus: case report and literature review. World J Gastroenterol (2013) 19(33):5586-5589.

[7] Howells L, Liasis L, Demosthenous M. Gallstone coleus: a rare relation of gallstone ileus. Int J Surg Res (2016) 2(4):28 31 .

[8] Mallipeddi MK, Pappas TN, Shapiro ML, Scarborough JE. Gallstone ileus: revisiting surgical outcomes using National Surgical Quality Improvement Program data. J Surg Res (2013)184(1): 84-88.

[9] Martín-Pérez J, Delgado-Plasencia L, Bravo-Gutiérrez A, Burillo-Putze G, Martínez-Riera A, Alarcó-Hernández A, Medina-Arana V. Gallstone ileus as a cause of acute abdomen. Importance of early diagnosis for surgical treatment. Cir Esp 9 (2013) 1(8):485-489.

[10] Nuño-Guzmán CM, Marín-Contreras ME, Figueroa-Sánchez M, Corona JL. Gallstone ileus, clinical presentation, diagnostic and treatment approach. World J Gastrointest Surg (2016) 8(1):65-76.

[11] Rigler L. G., Borman C. N., Noble J. F.: Gallstone obstruction: pathogenesis and roentgen manifestations. JAMA, 1941, 117: 1753-1759.
[12] Chou J.-W., Hsu C.-H., Liao K.-F., et al.: Gallstone ileus: Report of two cases and review of the literature. World J Gastroenterol, 2007, 13: 1295-1298.

[13] Swift SE, Spencer JA. Gallstone ileus: CT findings. Clin Radiol (1998) 53(6):451-454.

[14] Liang X, Li W, Zhao B, Zhang L, Cheng Y (2015) Comparative analysis of MDCT and MRI in diagnosing chronic gallstone perforation and ileus. Eur $\mathrm{J}$ Radiol 84(10):1835-1842.

[15] Furukawa A, Yamasaki M, Furuichi K, Yokoyama K, Nagata T, Takahashi M, Murata K, Sakamoto T. Helical CT in the diagnosis of small bowel obstruction. Radiographics (2001) 21(2):341-355.

[16] Barakos JA, Ralls PW, Lapin SA, Johnson MB, Radin DR, Colletti PM, Boswell WD Jr, Halls JM Cholelithiasis: evaluation with CT. Radiology (1987) 162(2):415-418.

[17] Yu C. Y., Lin C. C., Shyu R. Y., et al.: Value of CT in the diagnosis and management of gallstone ileus. World $\mathrm{J}$ Gastroenterol, 2005, 11: 2142- 2147

[18] Ihara E., Ochiai T., Yamamoto K., et al.: A case of gallstone ileus with a spontaneous evacuation. Am J Gastro -enterol, 2002, 97: 1259-1260.

[19] Lobo D. N., Jobling J. C., Balfour T. W.: Gallstone ileus: diagnostic pitfalls and therapeutic successes. J Clin Gastroenterol, 2000, 30: 72-76. 